\title{
Severe apnoeas following immunisation in premature infants
}

\author{
M H Slack, D Schapira
}

\begin{abstract}
Four premature infants developed apnoeas severe enough to warrant resuscitation after immunisation with diphtheria, pertussis, and tetanus (DPT), and Haemophilus influenzae B (Hib). One required re-intubation and ventilation.

Although apnoeas after immunisation are recognised, they are not well documented. It is time for further research to elucidate the best time to immunise such infants.

(Arch Dis Child Fetal Neonatal Ed 1999;81:F67-F68)
\end{abstract}

Keywords: immunisations; apnoeas; prematurity

\section{Case reports}

Four previously stable premature infants, born under 30 weeks of gestation, developed profound apnoeic and bradycardic episodes within 24 hours of being immunised with diphtheria/tetanus/pertussis and Haemophilus influenzae B vaccines.

Case 1 was a boy born at 29 weeks of gestation, weighing $1070 \mathrm{~g}$, and ventilated for three days. His cerebral ultrasound scans were normal and his course unremarkable. He had no apnoeas and bradycardias for 13 days before immunisation. He received his first course of DTP and Hib at a corrected age of $36+4$ weeks. Within a few hours of immunisation he had multiple profound apnoeas and bradycardias and required intubation and ventilation. $\mathrm{He}$ quickly improved and was extubated after 36 hours. A full septic screen including a nasopharyngeal aspirate for respiratory syncytial virus was negative. He underwent his second and third course at term and 6 weeks without event.

Case 2 was a girl born at 26 weeks of gestation, weighing $740 \mathrm{~g}$, and ventilated for 20 days. She sustained a pulmonary haemorrhage and required ligation of a patent ductus arteriosus (PDA). Her cerebral ultrasound scans were normal. Before vaccination she had only the occasional apnoea and bradycardia that required stimulation. At a corrected gestational age of 40 weeks she was immunised with her first course of DTP and Hib. Within 24 hours she was having multiple profound apnoeic episodes requiring resuscitation with bag and mask ventilation. These settled over the following day. The second and third courses were given a year later without any adverse effects.

Case 3 was a boy born at 26 weeks of gestation, weighing $750 \mathrm{~g}$, and ventilated for 30 days. A PDA was closed with indomethacin. His cerebral ultrasound scans were normal. Apnoeas and bradycardias of prematurity had stopped 11 days before immunisation. At a corrected gestational age of 39 weeks he was immunised with his first course of DTP and Hib. Within 48 hours he went off his feeds, became tachypnoiec, and sustained several apnoeic episodes with recorded drops in $\mathrm{O}_{2}$ saturations. These required treatment with facial $\mathrm{O}_{2}$ and suction, but settled over 24 hours. His second and third immunisations given a month apart were uneventful.

Case 4 was a boy born at $27+6$ weeks of gestational age, and weighing $900 \mathrm{~g}$. He was ventilated for 5 weeks, developing recurrent pneumothoraces. A PDA was closed with indomethacin. He developed intraventricular haemorrhages and haemorrhagic hydrocephalus requiring a ventriculo-peritoneal shunt insertion. In the weeks leading up to immunisation he had only the occasional apnoea and bradycardia. He was immunised with his first course of DTP and $\mathrm{Hib}$ at a corrected gestational age of 42 weeks. Within 12 hours he was having multiple profound apnoeas, bradycardias, and desaturations, requiring resuscitation with bag and mask ventilation. These settled the following day. Six weeks later he received his second course of DTP and Hib without any adverse effect.

\section{Discussion}

It is common practice in neonatal units to immunise premature infants with DTP and Hib at around eight weeks after delivery. Although the occurrence of apnoeic episodes in premature babies immunised at eight weeks is recognised, it has not been well documented in the UK. Indeed, the most recent UK study did not look at apnoea as an adverse event. ${ }^{1}$ In Australia and North America one retrospective and two prospective studies, ${ }^{2-4}$ estimated the risk of apnoea after immunisation in premature infants at between 17-23\%. Most episodes were clinically insignificant. Some episodes, however, may be profound enough to require respiratory support. The presumed cause is the pertussis component.

Two studies ${ }^{2}{ }^{4}$ suggested that those infants more at risk of apnoea tend to be of a lower gestation and birthweight, require ventilation for longer, and are more likely to have chronic lung disease. One study ${ }^{3}$ showed no significant difference between those infants who had apnoeas and those who did not.

It is clear from our experience that some premature infants are at risk of life threatening apnoea after immunisation. During the period in which these cases occurred, we immunised a total of 51 infants of a similar gestational age. The risk of severe apnoea may, therefore, be as high as $8 \%$ in those infants born at less than 30 weeks of gestation. We believe that further, larger studies are needed to show the exact risk to these smaller infants. It would be interesting to know if the risk could be reduced by using the 
acellular pertussis vaccine. Depending on the outcome of these trials, it may be necessary to delay immunisation in extremely low birthweight infants.

1 Ramsay ME, Miller E, Ashworth LAE, Coleman TJ, Rush $\mathrm{M}$, Waight PA. Adverse events and antibody response to accelerated immunisation in term and preterm infants. Arch Dis Child 1995;72:230-2.
2 Botham SJ, Isaacs D. Incidence of apnoea and bradycardia in preterm infants following triple antigen immunisation. $\mathcal{F}$ Paediatr Child Health 1994;30:533-5.

3 Botham SJ, Isaacs D, Henderson-Smart DJ. Incidence of apnoea and bradycardia in preterm infants following DTPw and Hib immunisation: A prospective study. $\mathcal{F}$ Paediatr Child Health 1997;33:418-21.

4 Sanchez PJ, Laptook AR, Fisher L, Sumner J, Risser RC, Perlman J M. Apnea after immunisation of preterm infants. $\mathcal{F}$ Pediatr 1997;130:746-51. 